\title{
Определение термодинамических параметров в областях фазовых переходов в $\mathrm{Cu}_{1.95} \mathrm{Ni}_{0.05} \mathrm{~S}$
}

\author{
(C) Ф.Ф. Алиев ${ }^{1}$, Г.А. Гасанов ${ }^{2}$, А.Г. Рзаева ${ }^{3}$, М.Б. Джафраров ${ }^{1}$, Г.М. Дамиров ${ }^{1,4}$ \\ ${ }^{1}$ Институт фризики Национальной академии наук Азербайджана, \\ AZ-1143 Баку, Азербайджан \\ ${ }^{2}$ Академия Службы государственной безопасности им. Гейдара Алиева Азербайджанской Республики \\ AZ-1029 Баку, Азербайджан \\ ${ }^{3}$ Азербайджанский государственный педагогический университет, \\ AZ-1000 Баку, Азербайджан \\ ${ }^{4}$ Сумгаитский государственный университет, \\ AZ-5008 Сумгаит, Азербайджан \\ E-mail: farzali@physics.ab.az.
}

(Получена 17 января 2017 г. Принята к печати 23 января 2017 г.)

Анализированы данные рентгенодифрактометрического и дифференциально-термического анализов (ДТА) в области фазовых переходов. Обнаружено, что низкотемпературная ромбическая $\alpha$-фаза в области температур 370-390 K переходит в гексагональную $\beta$-фазу, а в области температур $740-765 \mathrm{~K}$ в кубическую $\gamma$-фазу по схеме: $\alpha \rightarrow \alpha+\beta \rightarrow \beta+\gamma \rightarrow \gamma$. По данным температурной зависимости дифференциального термического анализа выявлено, что $\alpha \rightarrow \beta$-переход сопровождается поглощением, а $\beta \rightarrow \gamma$ выделением тепла. Установлено, что оба перехода являются разрешенными и относятся к реконструктивному типу. Выявлено, что оба перехода происходят во флуктуационном объеме $\sim 10^{-20} \mathrm{~cm}^{3}$ и с температурными скоростями 0.11 и $0.08 \mathrm{~K}^{-1}$. Показано, что переход $\alpha \rightarrow \gamma$ сопровождается последовательной сменой структур, проходящих через промежуточную $\beta$-фазу, являющуюся несоразмерной относительно $\alpha$ - и $\gamma$-фаз.

DOI: $10.21883 /$ FTP.2018.01.45322.8519

\section{1. Введение}

При исследовании фазовых переходов (ФП) одним из важных аспектов является выявление взаимосвязи структурных и тепловых характеристик материала. Для определения этой связи необходимо исследовать физические свойства материала в температурной области ФП, позволяющие получить информацию о самом процессе ФП. Другой актуальный вопрос - определение распределения сосуществующих фаз в области ФП. Как известно, ФП, происходящие вследствие флуктуаций физического состояния вещества, обусловливают и все изменения физических свойств, происходящие в этой области. Феноменологическая теория размытых ФП [1] основывается на теории гетерофазовых флуктуаций, вводится функция включения фаз $L$, характеризующая распределение сосуществующих фаз в области ФП и ее производная по температуре $d L / d T$ (температурная скорость ФП). По существу функцией $L$ можно определить изменение всех термодинамических параметров системы, происходящих в области ФП.

Различные твердые растворы, обладающие высокими значениями термоэффективности [2,3] были получены замещением атомов меди атомами различных металлов в халкогенидах типа $\mathrm{Cu}_{2} X$ (где, $\left.X=\mathrm{S}, \mathrm{Se}, \mathrm{Te}\right)$. Вопервых, следует отметить, что $\mathrm{Cu}_{2} \mathrm{~S}$ имеет три полиморфические модификации [4,5]: первая -- орторомбическая $\alpha$-модификация, которая стабильна до $376.5 \mathrm{~K}$ и имеет элементарную ячейку с параметрами $a=14.90 \AA$, $b=27.28 \AA, c=13.41 \AA$ (пр. гр. Abm2); вторая - гекса- гональная $\beta$-модификация, которая стабильна в области $376.5-708 \mathrm{~K}$ и имеет элементарную ячейку с параметрами $a=3.89 \AA, c=6.68 \AA$; третья - кубическая модификация с периодом решетки $a=14.90 \AA$ (пр.гр. $F m \overline{3} m)$, которая существует при температурах выше $780 \mathrm{~K}$. Согласно [6], орторомбическая модификация имеет элементарную ячейку с параметрами $a=11.80 \AA, b=27.20 \AA$, $c=22.70 \AA$. В работе [7] указано, что $\mathrm{Cu}_{2} \mathrm{~S}$ при комнатной температуре имеет моноклинную решетку с параметрами $a=15.246 \AA, b=11.884 \AA, c=13.494 \AA$ и $\beta=116^{\circ} 35^{\prime}$ (пр.гр. $P 2_{1} / c$ ). В свете вышеизложенного представляет интерес синтезировать твердый раствор $\mathrm{Cu}_{2-x} M_{x} \mathrm{~S}$ (где, $M=\mathrm{Ni}, x=0.05$ ) и исследовать его структуру в широкой области температур.

Одним из характерных материалов, обладающих ФП, является твердый раствор $\mathrm{Cu}_{1.95} \mathrm{Ni}_{0.05}$. Проанализированы рентгенодифрактометрические и термодинамические параметры материала при ФП. Изучение различных структурных характеристик, взаимодействующих модификаций до ФП и в процессе ФП даст возможность выявить механизм их превращений.

\section{2. Получение образцов $\mathrm{Cu}_{1.95} \mathrm{Ni}_{0.05} \mathrm{~S}$}

Для синтеза $\mathrm{Cu}_{1.95} \mathrm{Ni}_{0.05} \mathrm{~S}$ использованы исходные элементы следующей чистоты: $\mathrm{Cu}$ - 99.998, Ni — 99.90 и $\mathrm{S}$ особой чистоты. Кварцевая ампула заполнялась исходными веществами в количествах, необходимых для данного состава. Ампула эвакуировалась до давления 
0.193 Па. Синтез проводился в интервале температур $800-1150^{\circ} \mathrm{C}$. Для гомогенизации синтезированный образец в течение 100 ч отжигался при температуре $800^{\circ} \mathrm{C}$. Для получения монокристаллических образцов $\mathrm{Cu}_{1.95} \mathrm{Ni}_{0.05} \mathrm{~S}$ была выбрана комбинация методов медленного охлаждения и метода Бриджмена.

\section{3. Экспериментальная часть}

Рентгенографические исследования проведены на порошковом дифрактометре типа D8ADVAVNCE фирмы „Bruker“ при режиме трубки 40 кВ, 40 мА (излучение $\mathrm{Cu} K_{\alpha} \lambda=1.5406 \AA$ ) в интервале температур $300<T<1400 \mathrm{~K}$ с использованием температурной камеры ТТК 450.

Дифракционные отражения записывались в интервале углов $0 \leq 20 \leq 80^{\circ}$. Идентификация данных, полученных при исследовании образцов низкотемпературной модификации, проведена по программе TOPAS. Кристаллографические параметры уточнены по программе EVA. Полученные результаты представлены в таблицах 1-3.

Расчет (см. табл. $1-3$ ) показывает, что $\alpha$-фаза при комнатной температуре имеет ромбическую решетку с параметрами $a=26.50 \AA, b=15.39 \AA, c=13.85 \AA$ и пространственную группу (пр.гр. Abm2). При температуре $T=(379 \pm 2) \mathrm{K}$ она превращается в гексагональную $\beta$-фазу с параметрами $a=3.96 \AA, c=6.78 \AA$, пр. гр. $P 63 / m m c$, а при $(750 \pm 2) \mathrm{K}$ переходит в кубическую $\gamma$-фазу с модификацией периодом $a=5.788$ и пр.гр. $F m \overline{3} m$.

При охлаждении образца до температуры $T=750 \mathrm{~K}$ число дифракционных отражений от $g$-фазы остается неизменным. При температуре $T=730 \mathrm{~K}$ образец становится двухфазным: $\sim 60 \%$ кубическим, $\sim 30 \%$ гексогональным. Только при температуре $\mathrm{T}=710 \mathrm{~K}$ происходит полное $\gamma \rightarrow \beta$ превращение. При дальнейшей понижении температуры до $T=450 \mathrm{~K} b$-фаза переходит в гексагональную модификацию и в интервале темпе-

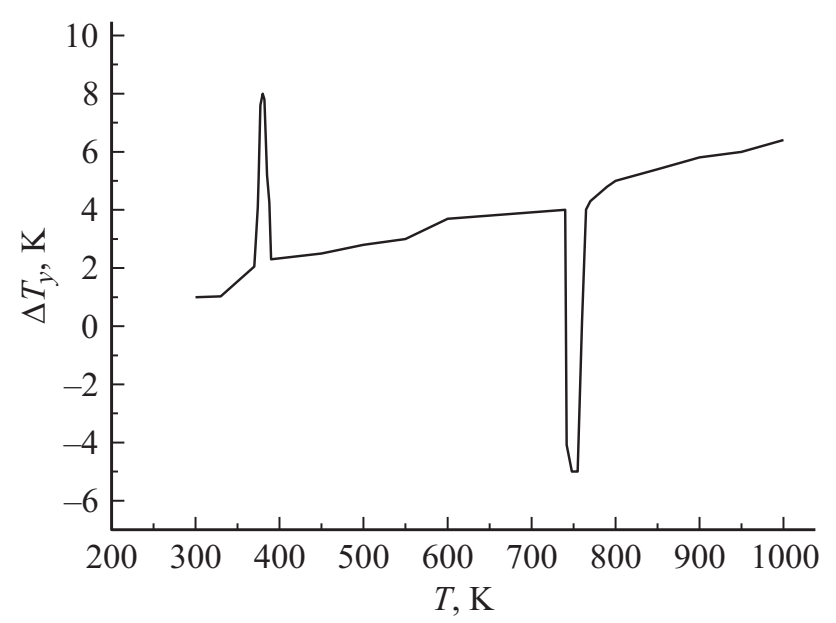

Рис. 1. Температурная зависимость $\Delta T_{y}$ в $\mathrm{cu}_{1.95} \mathrm{Ni}_{0.05} \mathrm{~S}$.
Таблица 1. Расчет дифрактограммы $\alpha$-модификации $\mathrm{Cu}_{1.95} \mathrm{Ni}_{0.05} \mathrm{~S}$ при $T=300 \mathrm{~K}$

\begin{tabular}{l|r|c|c|c|c|c|c|c}
\hline$d, \AA$ & $I$ & $h k l$ & $d, \AA$ & $I$ & $h k l$ & $d, \AA$ & $I$ & $h k l$ \\
\hline 4.243 & 6 & 222 & 2.659 & 25 & 224 & 1.949 & 30 & 116 \\
3.73 & 20 & 242 & 2.609 & 16 & 353 & 1.908 & 14 & 662 \\
$d, \AA$ & $I$ & $h k l$ & $d, \AA$ & $I$ & $h k l$ & $d, \AA$ & $I$ & $h k l$ \\
3.59 & 16 & 302 & 2.555 & 12 & 413 & 1.892 & 12 & 216 \\
3.41 & 6 & 203 & 2.524 & 40 & 423 & 1.876 & 90 & 2140 \\
3.31 & 25 & 180 & 2.469 & 45 & 433 & 1.797 & 14 & 593 \\
3.27 & 25 & 420 & 2.396 & 85 & 1111 & 1.787 & 14 & 691 \\
3.18 & 40 & 262 & 2.325 & 40 & 174 & 1.704 & 35 & 406 \\
3.15 & 30 & 421 & 2.237 & 25 & 205 & 1.657 & 12 & 286 \\
3.051 & 20 & 243 & 2.207 & 30 & 225 & 1.632 & 10 & 605 \\
2.942 & 45 & 091 & 2.117 & 12 & 444 & 1.528 & 25 & 486 \\
2.873 & 16 & 191 & 2.093 & 10 & 194 & 1.361 & 10 & 686 \\
2.755 & 16 & 134 & 2.005 & 14 & 345 & & & \\
2.718 & 55 & 044 & 1.977 & 100 & 2131 & & &
\end{tabular}

Таблица 2. Расчет дифрактограммы $\beta$-модификации $\mathrm{Cu}_{1.95} \mathrm{Ni}_{0.05} \mathrm{~S}$ при $T=379 \mathrm{~K}$

\begin{tabular}{c|r|c|c|c|c}
\hline$d, \AA$ & $I$ & $h k l$ & $d, \AA$ & $I$ & $h k l$ \\
\hline 3.388 & 5 & 002 & 1.361 & 2 & 203 \\
3.055 & 9 & 101 & 1.296 & 3 & 210 \\
2.401 & 88 & 102 & 1.281 & 11 & 114 \\
1.9805 & 100 & 110 & 1.273 & 4 & 211 \\
1.875 & 98 & 103 & 1.143 & 2 & 300 \\
1.706 & 45 & 200 & 1.122 & 3 & 213 \\
$d, \AA$ & $I$ & $h k l$ & $d, \AA$ & $I$ & $h k l$ \\
1.689 & 9 & 004 & 1.120 & 3 & 006 \\
1.661 & 10 & 201 & 1.082 & 5 & 302 \\
1.527 & 6 & 202 & 0.975 & 2 & 116 \\
1.509 & 3 & 104 & 0.949 & 2 & 222
\end{tabular}

Таблица 3. Расчет дифрактограммы $\gamma$-модификации $\mathrm{Cu}_{1.95} \mathrm{Ni}_{0.05} \mathrm{~S}$ при $T=756 \mathrm{~K}$

\begin{tabular}{c|r|r}
\hline$d, \AA$ & $I$ & $h k l$ \\
\hline 3.345 & 45 & 111 \\
2.894 & 18 & 200 \\
2.048 & 10 & 220 \\
1.746 & 25 & 311 \\
1.672 & 100 & 222 \\
1.1139 & 35 & 333
\end{tabular}

ратур 440-460 К одновременно существуют две фазы: $\sim 79 \%$ гексагональная и $\sim 20 \%$ ромбическая. Только при $T=350 \mathrm{~K}$ все дифракционные отражения ромбической $\alpha$-модификации полностью восстанавливаются.

Итак, рентгенографическим методом выявлено, что в образце $\mathrm{Cu}_{1.95} \mathrm{Ni0.05 \textrm {S }}$ в интервале температур 300-1400 K ФП носят энантиотропный характер и про- 
исходят по следующей схеме:

$$
\alpha \underset{350 \mathrm{~K}}{\stackrel{380 \mathrm{~K}}{\rightleftarrows}} \beta \underset{710 \mathrm{~K}}{\stackrel{750 \mathrm{~K}}{\rightleftarrows}} \gamma
$$

В исследуемом образце для выявления корреляции между рентгеноструктурными данными и тепловыми процессами проведены также аналогичные исследования методом дифференциально-термического анализа (ДТА) на приборе Perkin Elmer Sinintltaneons Thermal Analgiyzer, STA 600 (США). Образец нагревался со скоростью $5^{\circ} \mathrm{C} /$ мин. В качестве рабочего газа взят азот и его скорость составляла 20 мл./мин.

На основе ДТА выявлено, что в образце $\mathrm{Cu}_{1.95} \mathrm{Ni}_{0.05} \mathrm{~S}$ в интервале температур 370-390 K происходит поглощение, а при 740-775 К - выделение тепла (рис. 1).

\section{4. Методика определения термодинамических параметров в области фазовых переходов}

В последние 50 лет сильно развивалась теория физики размытых фазовых переходов [1,8-10]. В этих работах анализируется сосуществование каждой из фаз в области фазового перехода. Для выявления закономерности распределения массы в зависимости от температуры была использована функция включения $L(T)$ из теории размытых ФП конденсированных сред. Обозначив термодинамические потенциалы для $\alpha$ - и $\beta$-фаз через $\Phi_{\alpha}$ и $\Phi_{\beta}$, для области сосуществования фаз напишем

$$
\Phi(T)=\Phi_{\alpha}(T)-\Delta \Phi(T) L(T)
$$

где $\Delta \Phi(T)=\Phi_{\beta}(T)-\Phi_{\alpha}(T)$. Согласно теории размытых фазовых переходов (РФП), для функции включения имеет выражение [9]

$$
L(T)=\left\{1+\exp \left[a_{0}\left(T-T_{0}\right)\right]\right\}^{-1},
$$

где постоянная $a_{0}$, характеризующая степень размытия ФП, зависит от объема возможных фазовых флуктуаций, энергии и температуры ФП. Если учесть закон распределения масс от зависимости температуры в области ФП, тогда функция включения имеет вид

$$
L(T)=\frac{m_{\beta}(T)}{m_{\alpha}(T)+m_{\beta}(T)}=\left[1+\frac{m_{\alpha}}{m_{\beta}}(T)\right]^{-1},
$$

где $m_{\alpha}$ и $m_{\beta}$ - массы $\alpha$ - и $\beta$-фазы.

Из температурной зависимости $\ln \left(m_{\varepsilon} / m_{\beta}(T)\right)$ можно определить температуру $T_{0}$, где массы обоих фаз количественно равны. Из формулы (2) и (3) получим

$$
a_{0}=\frac{1}{T_{0}-T} \ln \left(\frac{m_{\alpha}}{m_{\beta}}\right)
$$

Если $a_{0}-$ некоторая постоянная, то множитель $\ln \left(\frac{m_{\alpha}}{m_{\beta}}\right)$ должен быть линейной функцией температурной

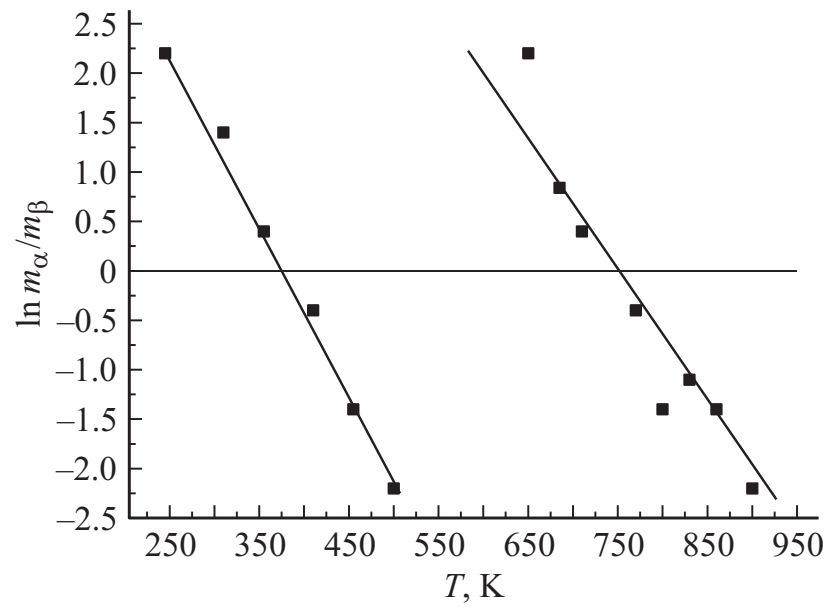

Рис. 2. Температурные зависимости распределения масс $\ln m_{\alpha} / m_{\beta}$ при фазовых переходах в $\mathrm{Cu}_{1.95} \mathrm{Ni}_{0.05} \mathrm{~S}$.

разности $\left(T_{0}-T\right)$ (рис. 2). Авторы [9] делали попытки определения этой функции на основе рентгеноструктурного анализа твердого тела. Они предполагали, что в узкой области сосуществования фаз температурное изменение интенсивностей рентгеновских отражений обусловлено количественным изменением фаз. Подобно этому $L(T)$ также можно определить по данным $\Delta T_{y}(T)$.

Для этого необходимо в области перехода добиться линейного изменения $\ln \left(\frac{m_{\alpha}}{m_{\beta}}\right)$ от температуры. Тогда для всего перехода интервал температур $\Delta T$ можно разбить на равные промежутки и соответствующие значения исследуемых эффектов отнести к предполагаемых фазам, например,

$$
\Delta T_{y}=\Delta T_{y, \alpha}\left(1-\frac{m_{\beta}}{m_{\alpha}}\right)+\Delta T_{y}, \beta\left(\frac{m_{\beta}}{m_{\alpha}}\right) .
$$

На рис. 2 представлены температурные зависимости $\ln y(T) y=\left(\frac{m_{\beta}}{m_{\alpha}}\right)$, где соответствующие массы определены из данных

$$
\Delta T_{y} \propto\left(\frac{m_{\beta}}{m_{\alpha}}\right)\left(m_{\alpha} \sim \Delta T_{y} ; m_{\beta} \sim \Delta T_{y, \beta}\right) .
$$

По точке пересечения прямой с осью абсцисс определены условные температуры $T_{0}$ обнаруженных ФП, где обе массы равны. Прямые на этой зависимости описываются выражением $y=\exp \left[-a_{0}\left(T-T_{0}\right)\right]$, где значения $a_{0}$, определенные из наклона прямых, являются температурной постоянной перехода. Во всех координатах прямые охватывают почти весь интервал ФП, что указывает на справедливость использованной методики - определения закона распределения массы от зависимости температуры, как $m_{\beta} / m_{\alpha}(T)$.

По данным $a_{0}$ и $T_{0}$ формулы (2) определены функции включения $L(T)$ и ее производные по тем- 
Таблица 4. Термодинамические параметры $\mathrm{Cu}_{1.95} \mathrm{Ni}_{0.05} \mathrm{~S}$ в области фазовых переходов

\begin{tabular}{l|c|c|c|c|c|c|c|c|c}
\hline Переход & $\begin{array}{c}T_{0}, \\
\mathrm{~K}\end{array}$ & $\begin{array}{c}a_{0}, \\
\mathrm{~K}^{-1}\end{array}$ & $\begin{array}{c}Q, \\
\text { кал/г }\end{array}$ & $\begin{array}{c}V_{\mathrm{ph}}, \\
\mathrm{cm}^{3}\end{array}$ & $\begin{array}{c}\Delta H, \\
\text { кал/моль }\end{array}$ & $\begin{array}{c}E_{a}, \\
\text { эВ }\end{array}$ & $\begin{array}{c}B, \\
\text { эВ }\end{array}$ & $\begin{array}{c}\Delta S, \\
\text { кал/моль } \cdot \mathrm{K}\end{array}$ & $\begin{array}{c}C_{p}, \\
\text { кал/моль } \cdot \mathrm{K}\end{array}$ \\
\hline$\alpha \rightarrow \beta$ & 380 & 0.44 & 34.8 & 4.6 & 764 & 0.18 & -0.10 & 2.01 & 100.1 \\
$\beta \rightarrow \gamma$ & 750 & 0.32 & 6.0 & 2.4 & 958 & 0.14 & -0.06 & 1.28 & 92.6
\end{tabular}

пературе $d L / d T$.

$$
\frac{d L}{d T}=\frac{a_{0}}{2} \frac{1}{1+\operatorname{ch}\left[a_{0}\left(T-T_{0}\right)\right]}
$$

выражающие температурные скорости фазового перехода. С помощью функции включения можно определить характер поведения и величину скачка различных термодинамических величин в области ФП.

Согласно теории гетерофазовых флуктуаций $[8,9]$, постоянная $a_{0}$ также определяется как $a_{0}=$ $=V_{\mathrm{ph} 0} Q \cdot k^{-1} T_{0}^{-2}$, где $V_{\mathrm{ph} 0}-$ объем фазовой флуктуации, $Q$ - количество выделения (или поглощения) тепла. Здесь величину $V_{\mathrm{ph} 0}$ можно рассматривать как минимальный объем, в котором происходит четкий ФП, или как объем частицы новой фазы внутри старой. Температурную зависимость $V_{\mathrm{ph}}(T)$ можно определить по формуле [11]

$$
V_{\mathrm{ph}}(T)=\frac{k T_{0}^{2} a_{0}}{Q} \frac{a_{0}}{2} \frac{1}{1+\operatorname{ch}\left[-a_{0}\left(T-T_{0}\right)\right]},
$$

Одни из важных термодинамических параметров характеризующих ФП, - энтропия превращения и удельная теплоемкость в условной точке $T_{0}$ могут быть определены из соотношения [12]

$$
\begin{gathered}
S=-\frac{\partial \Phi}{\partial T}=S_{1}+\Delta S L, \\
C_{p}=T \frac{\partial S}{\partial T}=C_{p 1}+\Delta C_{p} L+T \Delta S \frac{d L}{d T},
\end{gathered}
$$

где $S_{1}$ и $C_{p 1}$ - энтропия и удельная теплоемкость до начала $Ф П, \Delta S-$ энтропия превращения. Здесь, $\Delta C_{p}$ вычисляется по данным $Q, \Delta T, m\left(\Delta C_{p}=Q / m \Delta T_{1}\right)$, где для поглощения $\Delta T_{1}=T_{\text {fin }}-\left(T_{\text {pri }}+v t\right)$, а для выделения тепла $\Delta T_{1}=T_{\text {fin }}-\left(T_{\text {pri }}-v t\right), v$-скорость нагревания (или охлаждения), $t$ - время превращения. Изменение энтропии в области ФП может быть определено из соотношения $\Delta S=\Delta H / T_{0}$. Изменение энтальпии $\Delta H$ при ФП приравнивается к количеству тепла на один моль, вычисленному на основе ДТА в единицах кал/г (см. табл. 4).

Известно, что температурная зависимость удельной теплоемкости в области ФП определяется по формуле [11]

$$
C_{p}=C_{p 1}+\frac{a_{0} Q}{2 m} \frac{1}{1+\operatorname{ch}\left[\alpha_{0}\left(T-T_{0}\right)\right]} .
$$

Идентичный расчет также проведен для $\beta \rightarrow \gamma$-перехода. Полученные данные представлены на рис. 2-4 и в табл. 4.

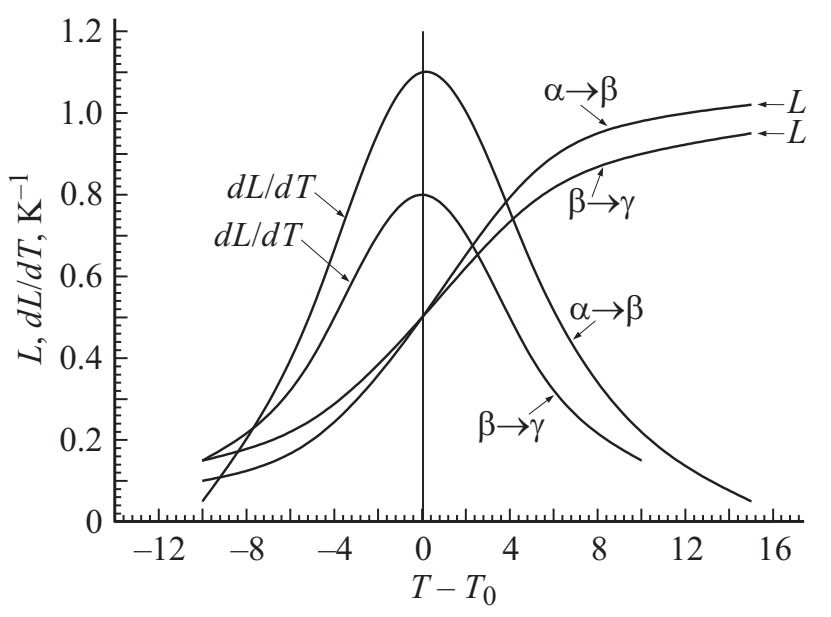

Рис. 3. Температурные зависимости функции включения $L$ и ее прозводной $d L / d T$ при фазовых переходах в $\mathrm{Cu}_{1.95} \mathrm{Ni}_{0.005} \mathrm{~S}$.

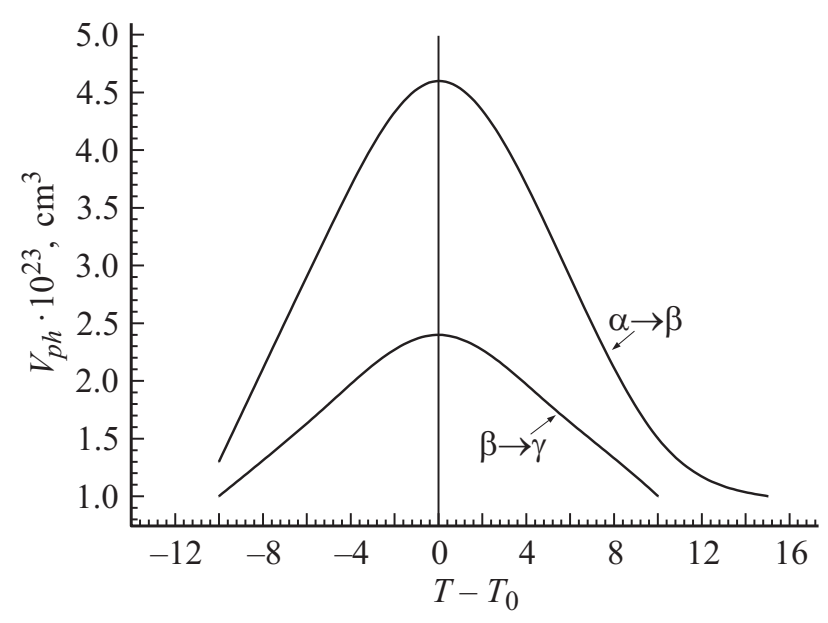

Рис. 4. Температурные зависимости флуктуации $V_{\mathrm{ph}}$ при фазовых переходах в $\mathrm{Cu}_{1.95} \mathrm{Ni}_{0.005} \mathrm{~S}$.

\section{5. Интерпретация результатов}

Известно, что структуры могут переходить одна в другую двумя способами. Если эти фазы обладают низкими симметриями, то переход должен происходить почти без искажения симметрии кристалла [13], при которой изменение внутренней энергии должно удовлетворять условию $\Delta S / R>\ln 2(R-$ универсальная газовая постоянная) [12]. Такие переходы принято называть ФП реконструктивного типа. 
Как показано в табл. $1-3$, в $\mathrm{Cu}_{1.95} \mathrm{Ni}_{0.05} \mathrm{~S}$ симметрии, обнаруженные в каждой фазе, сильно отличаются друг от друга. По длине химической связи $\mathrm{Cu} X$ (где $X-\mathrm{S}, \mathrm{Se}, \mathrm{Te})[14] \alpha \rightarrow \beta \rightarrow \gamma$-переходы преимущественно изменяются следующим образом: ковалентная $\rightarrow$ ковалентно-ионная $\rightarrow$ ионная. Следовательно, в образце $\mathrm{Cu}_{1.95} \mathrm{Ni}_{0.05} \mathrm{~S}$ симметрия и химические связи для каждых трех фаз сильно отличаются, и эти переходы удовлетворяют условию $\Delta S / R>\ln 2$ (см. табл. 4), так как эти переходы относятся к переходам реконструктивного типа. Из табл. 4 видно, что в $\mathrm{Cu}_{1.95} \mathrm{Ni}_{0.05} \mathrm{~S}$ изменение термодинамических параметров $\alpha \rightarrow \beta$-перехода намного меньше, чем при переходе $\beta \rightarrow \gamma$.

Это означает, что объем или же плотность кристалла в $\alpha$ - и $\beta$-фазах относительно ближе, чем в $\beta$ - и $\gamma$-фазах. Поэтому при $\alpha \rightarrow \beta$ переходе поглощается меньше тепла, чем выделяется при $\beta \rightarrow \gamma$. Это свидетельствует о том, что увеличивается внутренняя энергия, заключенная в структуре между атомами в решетках, затраченная на построение симметрии при переходах $\alpha \rightarrow \beta \rightarrow \gamma$.

Из перечисленных факторов вытекает, что в $\mathrm{Cu}_{1.95} \mathrm{Ni}_{0.05} \mathrm{~S}$ без разрыва существующих связей не могут происходить переходы $\alpha \rightarrow \beta \rightarrow \gamma$. Данный факт также проявляет себя при изменении $d L / d T$, так как для обоих переходов он меньше единицы. Как известно из [13], температурная скорость зависит от энергии активации $\left(E_{a}\right)$ ФП, где она определялась непосредственно из ДТА исследуемого вещества по формуле $E_{a}=R T_{\mathrm{pri}}^{2} / v t$, которая необходимо, для того чтобы переход стал разрешенным или неразрешенным [13]. Само разрешение перехода зависит от значения $B$ (высота энергетического барьера) [13]. Если $B>E_{a}$ переход неразрешен, а в случае $B<E_{a}$ переход становится разрешенным. В случае $\mathrm{Cu}_{1.95} \mathrm{Ni}_{0.05} \mathrm{~S} B$ можно оценить следующим образом: так как для обеих фаз удовлетворяется отношение $N_{\alpha} \propto \Delta H_{\alpha}, N_{\alpha}^{\prime} \propto \Delta H_{\alpha \rightarrow \beta}$, где $N_{\alpha}-$ концентрация атомов в $\alpha$-фазе, $N_{\alpha}^{\prime}-$ концентрация атомов, имеющих энергии, достаточные для преодоления энергетического барьера $B$, следовательно, $N_{\alpha}^{\prime}=N_{\alpha} \exp (-B / k T)$. Учтя данное предположение, получим отношение $N_{\alpha} / N_{\alpha}^{\prime} \propto \Delta H_{\alpha} / \Delta H_{\alpha \rightarrow \beta}$. Тогда можно определить $B$, т.е. $B=k T \ln \frac{\Delta H_{\alpha, \beta}}{\Delta H_{\alpha}}$, значение $\Delta H_{\alpha}$ до ФП взято из [16] (где $\Delta H_{\alpha}=17.2$ ккал/моль и $S_{\alpha}=25.5$ кал/моль $\cdot$ К). Подобные расчеты приведены и для $\beta \rightarrow \gamma$-перехода. Полученные значения для $B$ даны в табл. 4. Как видно из таблицы, для обоих ФП $B<E_{a}$, т. е. оба перехода являются разрешенными.

Можно предположить, что энергия $B$ также является причиной образования несоразмерной фазы. По данным рентгеноструктурного анализа видно, что при цикле $\alpha \leftrightarrow \beta \leftrightarrow \gamma$ возникает температурный гистерезис. Обычно температурный гистерезис связан с наличием несоразмерной новой фазы в старой фазе [16,17]. Роль несоразмерности при переходах связана с возможностью закрепления волны пространственной модуляции несоразмерной фазы на неоднородностях кристалла, хотя определенный вклад в закрепление вносит и дискретность решетки [17]. Это приводит к размытым ФП, которые обусловливаются запаздыванием превращения выше приведенной структуры и ее свойств в несоразмерной фазе с изменением температуры. Как видно из табл. 1-3, отношения постоянных структуры в $\alpha$ - и $\beta$-фазах (также в $\beta$ - и $\gamma$-фазах) являются иррациональными. Эта дает основания сказать, что переход $\alpha \rightarrow \gamma$ сопровождается последовательной сменой структур, проходящих через промежуточную $\beta$-фазу, являющуюся несоразмерной структурой по отношению к структуре $\alpha$ - и $\gamma$-фазы.

Одним из ключевых вопросов физики фазовых превращений является флуктуация физического состояния, возникающая при ФП. Считаем, что проведение расчетов объема флуктуации и ее распределение в области ФП могут быть полезными для исследования ФП в $\mathrm{Cu}_{1.95} \mathrm{Ni}_{0.005} \mathrm{~S}$. С этой точки зрения нами были построены температурные зависимости $V_{\text {рh }}$ для переходов $\alpha \rightarrow \beta$ и $\beta \rightarrow \gamma$ (рис. 4). Как видно из рисунка, для обоих переходов объем флуктуации достигает максимума в точке $T_{0}$ и асимптотично убывает по мере отдаления $T$ от $T_{0}$. В качестве источников, вызывающих флуктуации в кристалле $\mathrm{Cu}_{1.95} \mathrm{Ni}_{0.005} \mathrm{~S}$ принимали температурные неоднородности. Из зависимости $V_{\mathrm{ph}}(T)$ видно, что минимальный объем зародыша, играющего роль неоднородности, $\alpha$-фазы внутри $\beta$-фазы больше, чем $\beta$-фазы внутри $\gamma$-фазы. Следует отметить, что изменения внутренней энергии кристалла по мере включения зародышей $\alpha$-фазы в $\beta$-фазу и $\beta$-фазы в $\gamma$-фазу приводят к температурной неоднородности, так как причиной неоднородности является поликристаллическая структура кристалла. Причиной поликристалличности является сильное отличие структур в $\alpha$-, $\beta$ - и $\gamma$-фазах, так как при наличии $\alpha$-фазы в $\beta$-фазе и $\beta$-фазы в $\gamma$-фазе возникает поликристалличность в $\mathrm{Cu}_{1.95} \mathrm{Ni}_{0.005} \mathrm{~S}$.

Другим важным термодинамическим параметром, характеризующим тепловой эффект, является удельная теплоемкость материала. Из табл. 4 видно, что значения $Q, \Delta S$ и $\Delta H$ в точке $T_{0}$ сильно отличаются от нуля, что указывает на их принадлежность к ФП I рода. При ФП I рода температурная зависимость удельной теплоемкости должно изменяться скачкообразно. Как видно из рис. $5, C_{p}(T)$ качественно напоминает зависимость $\Delta T_{y}$ (рис. 1). В области $\alpha \rightarrow \beta$ и $\beta \rightarrow \gamma$ - переходов $C_{p}(T)$ проходит через пороговый максимум (рис. 5). Причину этого явления можно объяснить следующим образом: как видно из табл. 4 , в обоих ФП при точке $T_{0}$ объем фазовой флуктуации в получает оценку в порядке объема элементарной ячейки, т. е. $\approx 10^{-20} \mathrm{~cm}^{3}$. Эта дает основание предположить, что в начале, переход происходит в одной элементарной ячейке кристалла. За счет изменения тепловых колебаний угла связей, приводящих к образованию локальной тепловой энергии, происходит изменение этой энергии с температурой. С ростом температуры число таких обращенных областей возрастает быстро, и каждое локальное обращение способствует обращению соседних участков. При достижении температуры $T_{0}$ все углы кристалла должны колебаться с 


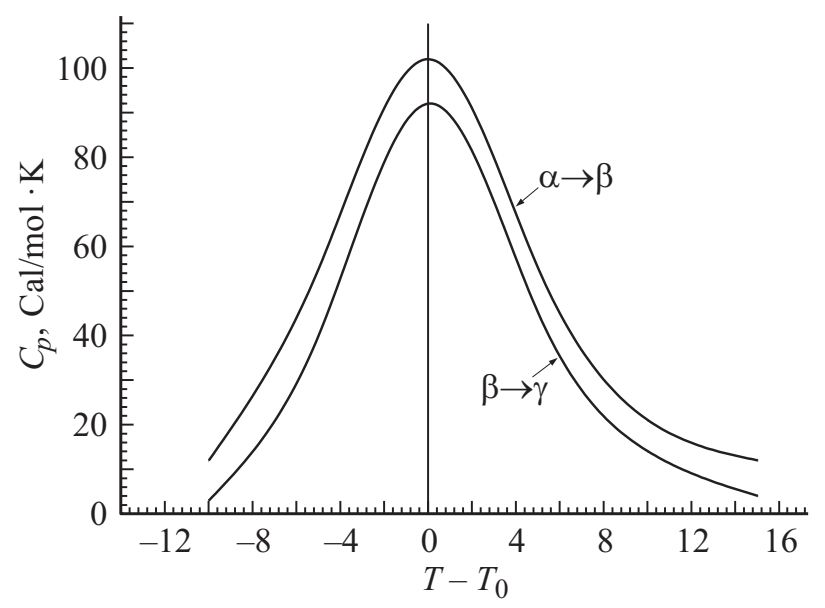

Рис. 5. Температурные зависимости удельной теплоемкости $C_{p}$ при фазовых переходах в $\mathrm{Cu}_{1.95} \mathrm{Ni}_{0.005} \mathrm{~S}$.

равными амплитудами (идет синхронизация колебаний узлов), благодаря чему передача энергии от одной стороны к другой не происходит. В итоге при $T=T_{0}$ $C_{p}(T)$ должен иметь разрыв, т.е. на зависимости $C_{p}(T)$ в точке $T_{0}$ должна появляться сингулярность. Однако в эксперименте $C_{p}(T)$ доходит до конечного значения. Причиной данного процесса являются флуктуации, образовывающиеся в следующих случаях: 1) образование зародышей новой фазы в старой фазе; 2) образование микроструктуры высокотемпературной $\beta$ - и $\gamma$-фазы при $\alpha \rightarrow \beta$ - и $\beta \rightarrow \gamma$-переходах; 3) образование неоднородностей кристалла и т.д. Каждый из перечисленных факторов могут являться причинами тепловых флуктуаций, приводящих к конечному значению $C_{p}(T)$ при $T_{0}$. При температурах $T>T_{0}$ начинается обратный процесс, приводящий к уменьшению $C_{p}(T)$. Подобные анализы приведены в работе [8], где автор показал, что величина температурной флуктуации в областях ФП определяется радиусом корреляции.

Эти данные позволяют заключить, что экстремумы на $\Delta T_{y}(T)$ полностью напоминают аналогичную зависимость $C_{p}(T)$. Из данных $\Delta T_{y}(T)$ и $C_{p}(T)$ также следует, что в $\mathrm{Cu}_{1.95} \mathrm{Ni}_{0.005} \mathrm{~S}$ ФП $\alpha \rightarrow \gamma$ сопровождается $Ф П$ $\alpha \rightarrow \beta$ и $\beta \rightarrow \gamma$, и они происходят по схеме

$$
\alpha \rightarrow \underset{370-390 \mathrm{~K}}{\alpha+\beta} \rightarrow \underset{740-765 \mathrm{~K}}{\gamma}
$$

Температуры $Ф П T_{0}$, определяющиеся из данных рентгеноструктурного анализа, чуть смещены в область низких температур по сравнению с данными, определяющимися по $\Delta T_{y}(T)$. Причиной тому могут быть тепловые флуктуации.

Интересные результаты, имеющие аналогию с нашими данными, получены в [18], где на основе калориметрических исследований $\mathrm{Cu}_{2-x} \mathrm{Se}$ установлено, что в стехиометрическом $\mathrm{Cu}_{2} \mathrm{Se}$ ФП при $414 \mathrm{~K}$ имеет резкий переход $\alpha \rightarrow \alpha+\beta \rightarrow \beta$, близкий по характеру к изотермическому ФП (I рода). По мере возрастания $x$ пики $Ф П$ $\alpha \rightarrow \beta$ размываются, температура перехода уменьшается до $291 \mathrm{~K}$ (при $x=0.3$ ). Начиная с $x=0.17-0.3$ на кривых дифференциального сканирующего калориметра (ДСК), кроме тепловых эффектов эвтектоидного превращения, наблюдается и эндотермический эффект (при $189 \mathrm{~K})$, который при увеличении скорости охлаждения $v_{\text {cool }}$ до $5 \mathrm{~K} /$ мин возрастает по абсолютной величине и смещается в сторону высоких температур $(257 \mathrm{~K})$, т.е. происходит почти перед $\alpha \rightarrow \beta$-переходом, а при уменьшении $v_{\text {cool }}$ до $0.2 \mathrm{~K} /$ мин вовсе исчезает. Эти переходы авторы связывают с неполным происхождением эвтектоидного превращения. Резюмируя полученные результаты, авторы заключают, что переход в состоянии суперионной проводимости (СИП) в $\mathrm{Cu}_{2-x} \mathrm{Se}$ является сложным превращением эвтектического типа. Появления СИП в $\beta$-фазе обусловлено разупорядочением катионной подрешетки соединения. Теоретически показано [19], что в суперионном состоянии, когда катионная решетка полностью разупорядочена, ФП переходу может предшествовать частичное упорядочение.

Известно, что все соединения типа $\mathrm{Cu} X$ (где $X-\mathrm{S}$, $\mathrm{Se}, \mathrm{Te})$ и твердые растворы, полученные на их основе, такие как $\mathrm{Cu}_{1-x} \mathrm{Ni}_{x} X$, относятся к числу СИП [19] и, в частности, в в $\mathrm{Cu}_{1.95} \mathrm{Ni}_{0.005} \mathrm{~S}$. Межузельные ионы $\mathrm{Cu}$ обладают большой подвижностью и они могут упорядочиваться до температуры ФП, причем небольшой избыток $\mathrm{Cu}$ ускоряет этот процесс.

\section{6. Заключение}

Весь комплекс экспериментальных исследований $\mathrm{Cu}_{1.95} \mathrm{Ni}_{0.005} \mathrm{~S}$ позволяет сделать вывод о том, что переход $\alpha \rightarrow \gamma$ сопровождается последовательной сменой структур, проходящих через промежуточную $\beta$-фазу, где $\beta$-фаза является несоразмерной по отношению к $\alpha$ - и $\gamma$-фазам. Считаем, что определение распределения отношения массы обеих структур и минимального объема флуктуации в ФП и их температурных зависимостей в области каждого обнаруженного ФП могут дать полезные сведения для исследования ФП в $\mathrm{Cu}_{2-x} \mathrm{Ni}_{x} \mathrm{~S}$ в целом. По мере отдаления от ФП в кристалле $\mathrm{Cu}_{1.95} \mathrm{Ni}_{0.005} \mathrm{~S}$ в качестве источников, вызывающих флуктуацию состояния фаз в области их сосуществования, являются температурные неоднородности, образующиеся за счет изменения внутренней энергии кристалла, а также неоднородности, обусловленные сильным различием структур $\alpha$-, $\beta$ - и $\gamma$-фаз и появлением в связи с этим поликристалличности в новых фазах $\mathrm{Cu}_{1.95} \mathrm{Ni}_{0.005} \mathrm{~S}$ в низкотемпературной области.

\section{Список литературы}

[1] Б.Н. Ролов. Размытые фазовые переходы (Рига, Зинашне, 1972).

[2] L. Cuangminq, T. Schulmeyer, J. Brotz, A. Klein, W. Jaedermann. Thin Sol. Films, 431, 472 (2003).

[3] T. Howard, Jr. Evans. Z. Kristallogr., 150, 299 (1979). 
[4] S. Djurle. Acta Chem. Scand., 12 (7), 1415 (1958).

[5] E.H. Roseboom. Econom. Geol., 64, 641 (1966).

[6] N. Alsen. Ged. Forening. i Stokholm. Forhand, 59, 111 (1931).

[7] T. Howard, Jr. Evams. Z. Kristallogr., 150/299 (1979).

[8] Б.Н. Ролов. Физические свойства размытых фазовых переходов (Рига, РГУ, 1974).

[9] Б.Н. Ролов. Изв. АН Латв. ССР. Сер. физ. и техн. наук, 4, 33 (1983).

[10] К.П. Мамедов, М.Ф. Гаджиев, З.Д. Нуриева. ДАН СССР, 231 (1), 94 (1976).

[11] С.А. Алиев, Ф.Ф. Алиев. ФТП, 42 (4), 404 (2006).

[12] Ф.Ф. Алиев, М.Б. Джафаров, А.А. Саддинова. ФТТ, 52 (10), 2019 (2010).

[13] М.Дж. Бюргер. Кристаллография, 16 (6), 1084 (1971).

[14] A.F. Wells. Structural inorganic chemistry (Oxford, Clarendon Press, 1986).

[15] Н.А. Алиева, К.А. Аскерова, С.Д. Багирова, И.Я. Алиев, 3.И. Сулейманов, Ф.М. Мустафаев, А.С. Аббасов. ДАН Азерб., 4 (3-4), 92 (1999).

[16] R.A. Abbasov. Rep. Prog. Phys., 45, 587 (1982).

[17] J.P. Jamet. Phase Trans., 11, 335 (1988).

[18] Н.Х. Абрикосов, В.Ф. Банкина, М.А. Коржуев, Г.К. Доменский, О.А. Теплов. ФТТ, 25 (10), 2911 (1983).

[19] Ю.Я. Гуревич, Г.В. Резник, Ю.И. Харкац. ФТТ, 20 (6), 1661 (1978).

Редактор Г.А. Оганесян

\section{Determination of thermodynamic parameters at the phase transition regions in $\mathrm{Cu}_{1.95} \mathrm{Ni}_{0.005} \mathrm{~S}$}

F.F. Aliev1 , H.A. Hasanov², A.Q. Rzaeva ${ }^{3}$, M.B. Jafarov ${ }^{1}$, G.M. Damirov ${ }^{1,4}$

${ }^{1}$ Institute of Physics National Academy of Sciences, AZ-1143 Baku, Azerbaijan

${ }^{2}$ Azarbaijan Academy of State Security Service named after Heydar Aliyev,

AZ-1029 Baku, Azerbaijan

${ }^{3}$ Azerbaijan State Pedagogical University,

AZ-1000 Baku, Azerbaijan

${ }^{4}$ Sumgait State University,

AZ-5008 Sumgait, Azerbaijan

Abstract $X$-ray difractometric and differential thermal analys (DTA) data in phase transitions region in $\mathrm{Cu}_{1.95} \mathrm{Ni}_{0.005} \mathrm{~S}$ have been analysed. DTA revealed that the low temperature rhombic $\alpha$-phase in $\mathrm{Cu}_{1.95} \mathrm{Ni}_{0.005} \mathrm{~S}$ transfers into the hexagonal $\beta$-phase in the temperature range of $370-390 \mathrm{~K}$ and it transfers to the cubic $\gamma$-phase in the temperature range of $740-765 \mathrm{~K}$ with the scheme:

$$
\alpha \rightarrow \underset{370-390 \mathrm{~K}}{\alpha+\beta} \rightarrow \underset{740-765 \mathrm{~K}}{\beta+\gamma} \rightarrow \gamma
$$

By differential thermal analysis data it has been established that $\alpha \rightarrow \beta$ transition is accompanied by absorption whereas $\beta \rightarrow \gamma$ transition is by heat exudation. It has been revealed that both transitions are allowed transitions; they relate to the reconstructive type, and take place in the fluctuation volume of approximately $10^{-20} \mathrm{sm}^{3}$ with temperature rates of 0.11 and $0.08 \mathrm{~K}^{-1}$. Study showed that $\alpha \rightarrow \gamma$ transition is accompanied by the sequent change in structures passing through the intermediate $\beta$-phase which is disproportionate to $\alpha$ - and $\beta$-phases. 